\title{
Philosophiques
}

\section{Kant, le matérialisme et la psychologie rationnelle}

\section{Denis Sauvé}

Volume 14, numéro 2, automne 1987

URI : https://id.erudit.org/iderudit/027013ar

DOI : https://doi.org/10.7202/027013ar

Aller au sommaire du numéro

Éditeur(s)

Société de philosophie du Québec

ISSN

0316-2923 (imprimé)

1492-1391 (numérique)

Découvrir la revue

Citer cet article

Sauvé, D. (1987). Kant, le matérialisme et la psychologie rationnelle. Philosophiques, 14(2), 227-261. https://doi.org/10.7202/027013ar

\section{Résumé de l'article}

Dans la Dialectique transcendantale de la Critique de la raison pure, Kant présente une discussion et une critique de la métaphysique de ses prédécesseurs rationalistes. Le premier chapitre de la Dialectique traite du dualisme cartésien et du matérialisme. Mon but est de déterminer quelle est sa position vis-à-vis de ces deux thèses opposées. Il pourrait sembler à première vue qu'en raison du caractère inconnaissable du sujet pensant, on ne peut selon lui se prononcer sur la vérité ou la fausseté de ni l'une ni l'autre de ces deux thèses. Ce que je me propose de montrer, cependant, c'est qu'il avance des arguments contre le dualisme cartésien et le matérialisme et qu'il les rejette tous les deux. Ces arguments découlent de la doctrine de l'idéalisme transcendantal, une des thèses centrales de son épistémologie.
Ce document est protégé par la loi sur le droit d'auteur. L'utilisation des services d'Érudit (y compris la reproduction) est assujettie à sa politique d'utilisation que vous pouvez consulter en ligne.

https://apropos.erudit.org/fr/usagers/politique-dutilisation/ 


\title{
ARTICLES
}

\section{KANT, LE MATÉRIALISME ET LA PSYCHOLOGIE RATIONNELLE}

\author{
par Denis Sauvé
}

RÉSumÉ. Dans la Dialectique transcendantale de la Critique de la raison pure, Kant présente une discussion et une critique de la métaphysique de ses prédécesseurs rationalistes. Le premier chapitre de la Dialectique traite du dualisme cartésien et du matérialisme. Mon but est de déterminer quelle est sa position vis-à-vis de ces deux thèses opposées. Il pourrait sembler à première vue qu'en raison du caractère inconnaissable du sujet pensant, on ne peut selon lui se prononcer sur la vérité ou la fausseté de ni l'une ni l'autre de ces deux thèses. Ce que je me propose de montrer, cependant, c'est qu'il avance des arguments contre le dualisme cartésien et le matérialisme et qu'il les rejette tous les deux. Ces arguments découlent de la doctrine de l'idéalisme transcendantal, une des thèses centrales de son épistémologie.

ABSTRACT. In the Transcendental Dialectic of the Critique of Pure Reason Kant discusses and criticizes traditional (or rationalist) metaphysics. In the first chapter of the Dialectic his discussion bears on Cartesian mind-body dualism and its opposite thesis of materialism. The aim of this paper is to determine the nature of his views concerning these two doctrines. At first sight it might seem that Kant's position is that the thinking self is unknowable and, consequently, that no decision can be made with respect to the truth or falsity of either dualism or materialism. What I wish to show, however, is that Kant argues against the two doctrines and that he rejects them both. These arguments derive from one of his central epistemological tenets, transcendental idealism.

On connaît surtout le chapitre de la première Critique dans lequel Kant traite des "paralogismes de la raison pure» pour les 
objections qu'il y oppose à la "psychologie rationnelle». Pour Kant, la psychologie rationnelle est cette doctrine due principalement à Descartes, mais qui a été reprise aussi par d'autres philosophes rationalistes, d'après laquelle le sujet pensant, la res cogitans, est une substance différente par essence de la substance matérielle. Ce sont les arguments sur lesquels s'appuie, selon lui, la psychologie rationnelle qu'il qualifie ici de " paralogismes » et son but, essentiellement, est de présenter une analyse de chacun de ces arguments et de montrer ensuite pourquoi ils ne sont pas concluants. Cependant, on note moins souvent que Kant fait aussi dans ce chapitre des remarques à propos du matérialisme, le point de vue qui consiste à soutenir au contraire qu'il n'y a aucune différence de nature entre le sujet pensant et la matière, et il est clair d'après ces remarques que le matérialisme soulève pour lui au moins autant de difficultés que le dualisme corps-esprit de Descartes. La question que je voudrais poser est celle-ci : quelle est au juste sa position vis-à-vis de ces deux thèses, l'une qui dit que le sujet est une substance immatérielle, c'est-à-dire le point de vue du « psychologue rationnel », ou du dualiste cartésien, et l'autre suivant laquelle c'est une substance matérielle, le point de vue du matérialiste ? 1

Il semble que l'un des résultats importants de sa discussion des paralogismes soit que l'on ne peut démontrer ni l'une ni l'autre des deux thèses opposées. La nature du sujet pensant appartient selon lui au domaine du "suprasensible»; le sujet «en soi», ce qu'il est en lui-même, demeure nécessairement inconnaissable et si, comme le psychologue rationnel ou le matérialiste, nous voulions dire quelque chose sur l'essence du " moi », nous ferions « un pas en dehors du monde sensible [et nous entrerions] dans le champ des noumènes ${ }^{2}$. Pourtant, d'un autre côté, même si on ne peut rien savoir sur sa nature, est-ce qu'il se peut que le moi soit une chose telle que la conçoivent les matérialistes ? Kant ne développe pas beaucoup ses remarques sur ce point, mais, il me semble, ce qu'il en dit suggère une réponse négative: il n'est pas possible,

1. Dans ce qui suit, toutes les citations de Kant sont tirées de la Critique de la raison pure. J'utilise les conventions habituelles pour les références. La première édition est signalée par la lettre $A$, la seconde par la lettre $B$, suivie du numéro de page tel qu'il est donné dans les Gesammelte Schriften publiés par l'Académie de Berlin (Berlin: G. Reimer, 1911, vol. llı er IV), suivi ensuite du numéro de page de la traduction de A. Tremesaygues et B. Pacaud (Paris : Presses Universitaires de France, 1968).

2. B $409 ; 287-288$. 
selon lui, que le moi soit une chose de même essence que la matière. Il est vrai qu'on ne peut rien dire, positivement, sur ce qu'il est « en soi », mais nous sommes au moins certains qu'il n'est pas un être matériel au sens où le soutiennent les matérialistes. Comme je l'expliquerai plus loin, il ne s'ensuit pas pour autant selon lui qu'il faudrait souscrire à la thèse de l'immatérialité «en soi» du sujet au sens de Descartes.

Pour défendre cette interprétation, je vais rappeler d'abord brièvement (section 1) comment Descartes caractérise l'essence du moi, c'est-à-dire en quel sens c'est pour lui une substance immatérielle. Ensuite, je résumerai quelques-unes des objections que fait Kant à l'adresse de cette doctrine et, finalement (section 2 ), j'exposerai, entre autres, ce qu'on pourrait appeler sa « réfutation » du matérialisme.

\section{I}

Dans les Méditations, Descartes décrit de deux façons différentes l'opposition de nature qui existe selon lui entre l'esprit et les corps. D'un côté, il faut, dit-il, se représenter l'esprit comme une substance dont l'essence est d'être une chose qui pense, tandis qu'un corps est une substance dont l'essence est d'occuper un espace ou d'être une chose étendue. Ce sont ces deux propriétés, la pensée et l'étendue, qu'il mentionne le plus souvent lorsqu'il veut caractériser les natures respectives du moi et de la matière. Mais, d'un autre côté, le moi n'est pas seulement une chose pensante, c'est aussi par essence une substance simple, c'est-à-dire une chose non composée et indivisible ; un corps, au contraire, est toujours composé et divisible. Le fait que l'âme ne soit pas composée et ne possède pas de parties implique qu'elle n'est pas sujette à la destruction, par décomposition ou par division, et c'est pourquoi elle est immortelle. Par contraste, un corps apparaît comme étant nécessairement divisible parce qu'il n'y a aucune partie de la substance étendue, même la plus petite qu'on puisse imaginer, qui ne puisse être divisée, au moins potentiellement, en parties encore plus petites; en ce sens, il n'existe pas d'atomes ou de parties insécables de la matière. Il s'ensuit pour Descartes une incompatibilité et une opposition de nature entre le moi et les choses matérielles. D'une part, la divisibilité est une propriété indissociable de l'essence des 
corps : il y aurait incohérence à dire que quelque chose occupant un espace, même une parcelle de matière extrêmement petite, ne possède pas de parties et n'est pas divisible. De l'autre, il n'y a rien qui puisse être à la fois simple et composé (ou divisible et indivisible): les deux propriétés sont incompatibles. Mais, si l'âme, à la différence des corps, est indivisible, alors, nécessairement, elle diffère par essence de la substance étendue. C'est donc une substance immatérielle 3 .

Lorsque Kant discute la question de la matérialité ou de l'immatérialité du sujet pensant, c'est dans les termes qu'il emprunte à la seconde de ces deux oppositions : soit le sujet est simple (ou indivisible), soit il est composé (ou divisible). La question de son immatérialité se réduit donc à celle de savoir s'il s'agit, comme le dit Descartes, d'une substance simple, par conséquent immatérielle, ou bien d'une substance composée comme le soutiennent les matérialistes. Pour cette raison, Kant donne le nom de «paralogisme de la simplicité » à l'argument qu'avance le psychologue rationnel à l'appui de la thèse de l'immatérialité.

Un autre argument important qu'il discute dans ce chapitre est le "paralogisme de la substantialité », un argument qui tend à montrer que le moi est une substance et non un attribut ou bien une propriété d'une substance. Pour le psychologue rationnel, le sujet pensant est une réalité substantielle au même titre que les corps, une «chose » à laquelle on doit rapporter les propriétés non physiques d'une personne, entre autres les propriétés dénotées par des verbes comme « penser », « douter », « affirmer », « imaginer », tandis que c'est au corps de la personne que l'on attribue l'ensemble de ses propriétés physiques. La thèse de la substantialité est fondamentale pour Descartes parce que si le moi n'était pas une substance, il deviendrait impossible de démontrer sa simplicité de nature et son immatérialité dans la mesure où il est évident qu'il doit exister quelque chose, une certaine substance, que l'on se représente comme indivisible et différente de la substance matérielle. Dans ce qui suit, je m'en tiendrai exclusivement à ce que dit Kant à propos de ces deux paralogismes, l'un concernant la substantialité du moi, l'autre sa simplicité et son immatérialité.

3. Cf. la sixième Méditation dans les Euvres philosophiques publiées par F. ALQuIÉ (Paris: Garnier, 1963-1973), tome II, p. 499; et également les Principes de la philosophie (deuxième partie, art. 20), tome III, p. 165-166. 
Pour comprendre quelles difficultés soulèvent selon lui les arguments du psychologue rationnel, prenons pour débuter le paralogisme de la substantialité. Il le formule comme suit:

Je puis dire de toute chose en général qu'elle est une substance, en tant que je la distingue de simples prédicats et de simples déterminations des choses. Or, dans toute pensée, notre moi est le sujet auquel les pensées sont inhérentes seulement en qualité de déterminations et ce moi ne peut pas être employé comme la détermination d'une autre chose. Chacun doit donc se considérer lui-même comme une substance et ses pensées seulement comme des accidents de son existence et des déterminations de son état. ${ }^{4}$

La première proposition rappelle la définition traditionnelle de la substance. Par le concept d'une substance, écrit Descartes dans les Principes, nous désignons «une chose qui existe en telle façon qu'elle n'a besoin que de soi-même pour exister », en quoi elle s'oppose aux qualités ou attributs, lesquels au contraire sont des «choses (...) de telle nature qu'elles ne peuvent exister sans quelques autres ", c'est-à-dire sans les substances auxquelles elles sont inhérentes ${ }^{5}$. De façon similaire, une substance, dit Kant, est un «être subsistant par [lui]-même " ${ }^{6}$, alors qu'on se représente les attributs comme de simples déterminations de la substance. Mais, comme on peut le lire dans la seconde Méditation, le moi, précisément, est cette chose «qui doute, qui conçoit, qui affirme, qui nie, qui veut, qui ne veut pas » et, dit Descartes, il n'y a «aucun de ces attributs (...) qu'on puisse dire être séparé de moi-même » ${ }^{7}$, car douter, concevoir et affirmer présupposent l'existence de ce qui doute, conçoit ou affirme, et aucune de ces propriétés ne peut être séparée du moi et être considérée comme une chose pouvant subsister par elle-même. Entre le moi et les pensées, il y a donc la même relation que celle qui existe en général entre une substance et ses attributs. Le psychologue rationnel en conclut que le moi est une substance et les pensées seulement des déterminations attachées à cette substance.

On trouve dans le commentaire que fait Kant de cet argument un certain nombre de remarques critiques différentes. Je vais

\footnotetext{
4. A. $348 ; 283$, trad. $\bmod$

5. Principes de la philosophie (première partie, art. 51), ouv. cit., p. 122

6. B $407 ; 284$.

7. Deuxième Méditation, ouv. cit., p. 420-421.
} 
résumer les trois remarques les plus importantes. En premier lieu, Kant se demande à quoi on reconnaît, concrètement, qu'une « chose » est une substance et non pas plutôt un attribut ou bien une propriété d'une substance. Il existe, dit-il, un critère empirique de la substantialité, et ce critère est la permanence : «nous sommes obligés de prendre pour principe la permanence d'un objet donné tiré de l'expérience si nous voulons lui appliquer le concept d'une substance tel qu'on peut l'employer empiriquement ${ }^{8}$. D'après ce critère, pour savoir ce qui signale la présence d'une chose substantielle dans une intuition empirique, on doit rechercher ce qu'il y a dans cette intuition de permanent et de constant, alors que les attributs de la chose se reconnaissent aux déterminations variables et changeantes de l'intuition. C'est aussi le seul usage qu'il est permis de faire du concept d'une substance: "[pour] que ce concept désigne (...) un objet qui puisse être donné (...), il faut qu'il ait pour fondement une intuition constante comme condition indispensable de [sa] réalité objective " ${ }^{9}$; "sans la condition de l'intuition sensible », le concept n'est « que d'un usage transcendantal, c'est-àdire d'aucun usage ${ }^{10}$. Dans le cas du moi, cependant, Kant soutient qu'on ne peut «démontrer une pareille permanence par une observation certaine ${ }^{11}$. Il est vrai que, d'une certaine façon, le moi "reparaît toujours dans toute pensée» 12 en ce sens que, comme le dit Descartes, il est évident que c'est moi qui doute, qui conçois ou qui imagine; mais lorsque par le «sens interne» (ou $l$ '« intuition intérieure ») une personne prend conscience par introspection de ses propres pensées, elle ne peut y découvrir une intuition de son moi, une "intuition fixe et permanente où se succéderaient (en tant que variables) les pensées ${ }^{13}$ car, dit Kant, "dans ce que nous appelons l'âme, tout est dans un continuel écoulement et il n'y a rien de permanent " ${ }^{14}$. Si on ne trouve rien qui permet de faire la distinction entre ce qui demeure et ce qui change dans les représentations qui sont données par le sens interne, alors « il nous manque (...) la condition nécessaire pour

\footnotetext{
8. A $349 ; 283$.

9. B $412 ; 293$.

10. A $403 ; 325$

11. A $350 ; 284$.

12. Ibid.

13. Ibid.

14. A $381 ; 308$
} 
appliquer au moi (...) le concept de la substance, c'est-à-dire d'un sujet existant par lui-même ${ }^{15}$.

Une seconde remarque explique et complète en partie la première. Par le sens interne, d'après la première remarque, nous ne trouvons rien en nous-mêmes de permanent ou de constant. S'il pouvait y avoir quelque chose comme une perception intérieure de ce qu'on nomme le « moi », une perception que chacun posséderait de la chose qu'il est lui-même, elle se présenterait, présumément, comme une sorte d'intuition persistante et durable à travers la succession continuelle des pensées. Or, justement, il se trouve qu' «[à la] représentation [du moi] n'est pas liée la moindre intuition qui la distinguerait de tous les autres objets de l'intuition ${ }^{16}$. Mais pourquoi? Le fait qu'il ne peut y avoir une auto-intuition du moi découle du caractère inobservable et non représentable du sujet par lui-même. En effet, comme le dit Kant dans la déduction transcendantale des catégories, le moi «forme le corrélatif de toutes [les] représentations $\gg{ }^{17}$. Si tel est le cas, alors il ne peut être pour lui-même une représentation parmi d'autres représentations ou bien un objet qu'il se représente parmi d'autres objets représentés ; il est plutôt, en tant que «sujet connaissant », ce qui parvient à une connaissance des objets au moyen de ces représentations. La raison pour laquelle, donc, le moi ne peut s'intuitionner ni se rendre présent à lui-même comme un objet, c'est que «je ne saurais connaître comme objet cela même [le sujet] qu'il me faut supposer pour connaître en général un objet ${ }^{18}$. Il s'ensuit que «nous n'avons ni ne pouvons avoir la moindre connaissance [de ce qu'est le moi] ${ }^{19}$. Si le sujet pensant ne peut être un objet de représentation pour lui-même, alors il n'est pas possible de dire quel genre d'entité est cette chose: "Par ce "moi", par cet "il" ou par ce quelque chose qui pense, on ne se représente rien de plus qu'un sujet transcendantal des pensées $=\mathrm{X}(\ldots)$ dont nous ne pouvons jamais avoir, séparément, le moindre concept ${ }^{20}$.

15. B $413 ; 293$.

16. A $350 ; 284$

17. A $123 ; 138$.

18. A $402 ; 324$

19. A $350 ; 284$.

20. A $346=$ B $404 ; 281$, trad. mod. 
La troisième remarque, finalement, ne constitue pas à proprement parler une objection au paralogisme de la substantialité, mais elle donne une autre raison de tenir la nature du moi pour quelque chose d'entièrement inconnaissable. Dans le commentaire du paralogisme, on lit entre autres l'affirmation suivante: "nous n'avons aucune connaissance du sujet en soi qui est à la base du moi comme de toutes les pensées en qualité de substrat ${ }^{21}$. Kant fait allusion ici à l'idée qu'il a défendue dans l'Esthétique transcendantale et ensuite à nouveau dans l'Analytique d'après laquelle il faut faire la distinction entre une chose telle qu'elle est en soi et cette même chose telle qu'elle nous apparaît en tant que "phénomène », une distinction qui est valable non seulement pour les objets des sens externes (ou les «objets extérieurs »), mais aussi pour l'objet du sens interne, le moi considéré comme le sujet des pensées ou, si le moi n'était pas une substance, pour la chose qui se trouve à la base du moi en même temps que des pensées. En effet, selon lui, nous nous représentons les choses extérieures comme un ensemble d'objets situés dans l'espace et dans le temps ; mais cette propriété qu'ils ont d'être situables dans un cadre spatio-temporel dépend essentiellement de la manière dont ils apparaissent au sujet à travers ses «formes» a priori que sont l'espace et le temps et ce n'est pas en eux-mêmes qu'ils possèdent cette propriété. De la même façon, le sujet pensant se connaît par les représentations qui lui sont données par le sens interne, dont la forme a priori est le temps, c'est-à-dire par les pensées, les intentions, les sentiments, et toutes les autres représentations qu'il s'attribue comme des propriétés de son moi, mais ces représentations ne lui font pas plus connaître ce qu'il est en soi que les représentations des sens externes ne lui apprennent ce que sont les objets extérieurs en euxmêmes. En ce sens, on peut dire que «L'objet transcendantal est également inconnu, qu'il s'agisse aussi bien de l'intuition interne que de l'intuition extérieure ${ }^{22}$. Et la conséquence, à nouveau, est qu'il n'est pas possible de savoir quel genre de chose est le sujet des pensées. Si «nous ne connaissons notre propre sujet que comme phénomène et non dans ce qu'il est en soi ${ }^{23}$, alors nous n'avons aucune idée de ce qu'il est et il n'y a rien que nous puissions dire sur son essence.

21. A $350 ; 284$.

22. A $372 ; 302$.

23. B $156 ; 135$. 
Il est fréquent dans le chapitre des paralogismes que Kant s'exprime comme si le moi était l'«objet» du sens interne de la même manière que sont donnés au sujet par le sens externe les objets extérieurs représentés dans la forme a priori de l'espace. Ce parallélisme entre la connaissance de soi par le sens interne et la connaissance des objets extérieurs par le sens externe contredit apparemment l'idée d'après laquelle le moi ne peut être un objet pour lui-même et qu'il n'existe aucune intuition du moi comme sujet des pensées. Mais la difficulté n'est peut-être qu'apparente. Kant, en effet, pourrait répondre de la façon suivante. Par l'intuition interne, le sujet possède la connaissance de lui-même (ou la connaissance de soi au sens habituel du terme) parce qu'il a conscience de façon immédiate et sans inférence du contenu de ses propres pensées, de ses sensations, de ses sentiments, ou de ses intentions ; une telle connaissance se manifeste, entre autres, par des propositions formulées à la première personne comme «J'ai telle ou telle pensée » ou bien «J'ai telle ou telle sensation ». Mais, bien qu'il s'attribue spontanément à lui-même ses pensées et toutes ses autres représentations, le sujet n'est pas pour autant « informé » de ce qu'il a ces pensées sur la base d'une observation de son propre moi (comme quelqu'un peut savoir qu'il a une tache d'encre sur la main parce qu'il a observé sa main). Il semble plutôt que pour Kant le moi «perçoive » auto-réflexivement ses pensées et non le sujet des pensées : il a l'« intuition » de ses représentations seulement (de ses intentions, sentiments, etc.) et non pas du moi lui-même qui a ces représentations. D'une certaine façon, il est vrai de dire que le moi constitue l'«objet» du sens interne, mais seulement en ce sens que c'est de ses pensées qu'il a connaissance par l'intuition intérieure, et non pas au sens où il pourrait y avoir une intuition empirique du moi semblable à l'intuition par laquelle nous sont donnés les objets dans l'espace.

Un autre point qui aurait besoin d'être élucidé concerne la distinction entre le sujet comme phénomène et le sujet comme "chose en soi». Kant écrit ceci : je n'ai «aucune connaissance de moi tel que je suis, mais je me connais simplement tel que je m'apparais à moi-même ${ }^{24}$. Si le moi se connaît seulement comme phénomène et non tel qu'il est en soi, dans ce qui définit

24. B $158 ; 136$. 
son essence, alors on pourrait se demander de quelle manière, plus précisément, il s'apparaît à lui-même. Les objets extérieurs nous apparaissent sous l'aspect de choses dotées de propriétés spatiales : un objet possède une forme, des dimensions et il entretient des relations spatiales avec d'autres objets. Mais sous quel aspect le moi s'apparaît-il à lui-même? Une réponse possible consisterait à dire que ce n'est pas à proprement parler le moi mais plutôt les pensées qui apparaissent au sujet, et ses pensées lui sont données par le sens interne comme des événements qui se succèdent dans le temps, par contraste avec les objets du sens externe, lesquels sont représentés à la fois dans la forme a priori de l'espace et dans la forme a priori du temps. Comme il l'écrit dans l'Esthétique, "Le temps n'est autre chose que la forme du sens interne, c'est-à-dire de l'intuition de nous-mêmes et de notre état intérieur (...). [Le temps] détermine le rapport des représentations dans notre état interne ${ }^{25}$. En ce sens, ce sont les pensées ou, en général, les représentations qui ont entre elles des rapports de temps (une pensée précède, une autre suit) et le temps est la « forme » propre aux représentations. Cependant, la difficulté est que, pour lui, c'est le moi que nous ne pouvons connaître tel qu'il est en soi et non les pensées, et il semble que c'est de la nature du sujet des pensées tel qu'il est en soi qu'il est ici question plutôt que de la nature des pensées elles-mêmes comme représentations. Il existe un passage du chapitre des paralogismes où il suggère une réponse différente : le moi, dit-il, s'apparaît, en tant que phénomène, comme une chose dont la seule propriété est d'avoir des pensées, ces pensées dont il a immédiatement conscience dans la connaissance qu'il a de luimême. Si les objets du sens externe nous apparaissent comme juxtaposés dans l'espace et comme ayant une forme et des dimensions, le moi s'apparaît à lui-même sous l'aspect d'une chose qui pense ; et de même que les objets extérieurs ne sont pas en euxmêmes situés dans l'espace, puisque l'espace n'existe pas en soi et qu'il est simplement la forme sous laquelle les choses extérieures nous apparaissent, le moi n'est pas non plus en soi, par nature, une chose pensante, il s'apparaît à lui-même seulement à travers le sens interne comme quelque chose qui a des pensées. Pourtant, il y a un autre endroit, toujours dans ce chapitre (et que je citerai plus loin) où Kant semble admettre au contraire la possibilité qu'il

25. А $33=$ B $49 ; 63$. 
appartienne à l'essence du moi d'être en soi une chose pensante, bien que selon lui on ne puisse savoir si telle est effectivement sa nature. Dans ce qui suit, je supposerai, provisoirement, qu'il est en effet possible pour lui que le moi soit une chose (en soi) pensante, et je reviendrai plus loin sur cette question.

Un point important à retenir de cette critique du paralogisme de la substantialité, il me semble, est qu'il ne faut pas en déduire que le sujet pensant $n$ 'est pas une substance. Il y a un seul critère pour établir que quelque chose est une substance et ce critère ne permet pas de savoir quel est le "mode d'existence " ${ }^{26} \mathrm{du}$ moi (être une propriété ou bien être le sujet des propriétés), mais tout ce qu'on peut en conclure c'est qu' ' il ne m'est pas possible de déterminer (...) la manière dont j'existe, si c'est comme substance ou comme accident $\gg{ }^{27}$. D'autre part, du fait que le sujet ne peut être un objet de représentation pour lui-même et qu'il ne se connaît pas tel qu'il est en soi, il ne suit pas qu'il ne pourrait pas être une chose dont la nature consiste dans la pensée. Il semble au contraire possible que la pensée, comme le soutient Descartes, appartienne à son essence. En somme, la difficulté dans le point de vue du psychologue rationnel n'est pas tellement qu'il est faux mais, plutôt, qu'il est impossible de prouver qu'il est vrai.

C'est ce qui ressort également de la critique que fait Kant du paralogisme de la simplicité. Par cet argument, selon lui, le psychologue rationnel ne réussit pas plus à prouver la simplicité du moi que, par le paralogisme de la substantialité, il ne réussit à prouver qu'il est une substance. Il est possible qu'il soit simple et immatériel, mais il se pourrait également, comme le soutiennent les matérialistes, qu'il y ait identité de nature entre le moi et la matière.

L'argument du paralogisme de la simplicité est, dans sa forme, une reductio ad absurdum. Le psychologue rationnel fait d'abord l'hypothèse que le moi est une substance composée. Mais étant donné que cette supposition entraîne, selon lui, une «contradiction», il en déduit qu'il est, non pas composé, mais simple. Kant énonce l'argument comme suit :

26. B $410 ; 289$.

27. В $420 ; 305$. 
Toute substance composée est un agrégat de plusieurs, et l'action d'un composé (...) est un agrégat de plusieurs actes ou accidents répartis entre la multitude des substances. Or, un effet qui résulte du concours de plusieurs substances agissantes est, sans doute, possible, quand cet effet est simplement extérieur (ainsi, par exemple, le mouvement d'un corps est le mouvement combiné de toutes ses parties); mais il en est autrement des pensées, comme accidents internes d'un être pensant. En effet, supposez que le composé pense; chacune de ses parties renfermerait alors une partie de la pensée et toutes ensemble seules contiendraient la pensée tout entière. Or, cela est contradictoire. En effet, puisque les représentations qui sont partagées entre différents êtres (par exemple, les mots particuliers d'un vers) ne constituent jamais une pensée entière (un vers), la pensée ne peut être inhérente à un composé comme tel. Elle n'est donc possible que dans une substance qui n'est pas un agrégat de plusieurs et qui, par conséquent, est absolument simple ${ }^{28}$.

Sans faire l'analyse du paralogisme, on pourrait le rendre plus explicite comme suit. Il existe une différence importante, d'après l'argument, entre une propriété d'une substance composée tel le mouvement et une pensée considérée comme une propriété ou un " accident interne » d'un être pensant. Le mouvement d'une substance composée se réduit au mouvement combiné de chacune des substances qui la composent. Mais est-ce qu'on peut imaginer une relation du même genre entre une pensée et le moi pensant dans l'hypothèse où celui-ci serait un agrégat de plusieurs substances? Si le moi était une chose composée, les parties de chacune de ses pensées seraient partagées entre ses différentes substances constituantes: une des substances "penserait 》 ou aurait conscience de l'une des parties de la pensée, une autre substance penserait une autre partie et ainsi de suite. Mais il ne pourrait pas résulter de là quelque chose comme une "pensée entière ", c'est-à-dire un complexe de plusieurs représentations réunies ensemble pour en former une seule, parce que chacune des parties du moi contiendrait une des représentations seulement dont est formée la pensée et il n'y en aurait aucune contenant toutes les représentations comme il serait nécessaire pour qu'il puisse en sortir une pensée complète. Le cas est le même que celui dans lequel différents individus ont connaissance de l'un des mots d'une certaine proposition (ou d'un vers, dans l'exemple du texte), mais où personne ne connaît simultanément tous les mots, de sorte qu'il n'y en a aucun qui peut

28. A. $351-352 ; 285-286$. 
savoir quelle pensée se trouve exprimée ou quel est le sens de l'ensemble de la proposition. En ce sens, l'argument se base sur la supposition que si le sujet pensant était un agrégat de plusieurs substances, on devrait regarder ses parties elles-mêmes comme des êtres pensants (probablement, bien que ce ne soit pas dit de façon explicite, parce que seul un être pensant est capable d'avoir des représentations). Si on fait cette supposition, alors il n'y a qu'un sujet, et non une pluralité de sujets, qui est susceptible de se représenter une pensée complète. En d'autres termes, «la pensée ne peut être inhérente à un composé comme tel», elle n'est possible que pour une substance pensante simple.

Le paralogisme de la simplicité n'est pas un argument que l'on peut trouver chez Descartes. Dans la sixième Méditation, Descartes écrit que si le moi était une substance composée, il n'y a que «les facultés de vouloir, de sentir, de concevoir, etc. » que l'on pourrait considérer comme ses parties. Or les facultés de l'esprit, dit-il, «ne peuvent pas proprement être dites ses parties : car le même esprit s'emploie tout entier à vouloir, et aussi tout entier à sentir, à concevoir etc. » Si ce n'est pas la faculté de vouloir qui veut, ou bien la faculté de sentir qui sent, mais le moi «tout entier », alors (si effectivement rien d'autre ne peut être une partie constitutive du moi), il s'ensuit que le moi ne possède aucune partie. Et il ajoute aussi: «Mais c'est tout le contraire dans les choses corporelles ou étendues : car il n'y en a pas une (...) que mon esprit ne divise fort facilement en plusieurs parties et par conséquent que je ne connaisse être divisible » ${ }^{29}$. Cette opposition entre le moi simple et la substance matérielle composée suffit à montrer, dit encore Descartes, « que l'esprit ou l'âme de l'homme est entièrement différente du corps ${ }^{30}$. Cependant, il est clair que cet argument à l'appui de la simplicité de la substance pensante est très différent du paralogisme discuté ici par Kant ${ }^{31}$.

29. Sixième Méditation, ouv. cit., p. 499.

30. Ibid.

31. Margaret WILSON, dans «Leibniz and Materialism» (Canadian Journal of Philosophy 3 [1974] ), soutient qu'il y a une similitude entre un argument avancé par Leibniz contre le matérialisme dans la Monadologie et le paralogisme de la simplicité. Elle écrit : «I propose that Leibniz's argument can plausibly be assimilated to a much more fully stated argument criticized by Kant in the «Paralogisms " section of the Critique of Pure Reason [i.e. le paralogisme de la simplicité] (and quite implausibiy associated in the Kant literature with the name of Descartes)" (p. 496). Plutôt que d'y voir un 
La critique du paralogisme se ramène essentiellement à deux remarques. Premièrement, Kant note que «le nervus probandi de cet argument se trouve dans la proposition: que plusieurs représentations doivent être contenues dans l'unité absolue du sujet pensant pour constituer une pensée ${ }^{32}$. Mais cette proposition n'exprime pas une vérité analytique : il n'y a aucune contradiction à supposer que c'est un moi composé plutôt qu'une substance simple qui pense l'ensemble des représentations dont est constituée une pensée complexe. En effet, "l'unité de la pensée qui se compose de plusieurs représentations est collective et peut se rapporter (...) à l'unité collective des substances qui la produisent (...) tout aussi bien qu'à l'unité [ou simplicité] absolue du sujet ${ }^{33}$. Donc, « d'après la règle de l'identité », ou le principe de contradiction, «il est (...) impossible de voir clairement la nécessité de la supposition d'une substance simple pour une pensée composée ${ }^{34}$. Puisque l'argument dépend de cette supposition, alors il n'arrive pas à prouver la simplicité du moi.

En deuxième lieu, Kant offre une explication de ce que nous avons tendance à voir dans le moi une substance simple et dénuée de parties. La représentation que nous nous faisons du moi est celle d'« un quelque chose en général », donc d'un sujet qui n'est désigné "que d'une manière transcendantale, sans qu'on en remarque la moindre propriété ${ }^{35}$. Or cette représentation du moi conçu seulement comme le substrat des pensées «doit être absolument simple par le fait même qu'on ne détermine rien [du moi], rien ne pouvant assurément être représenté plus simplement (einfacherer) que par le concept d'un simple (bloss) quelque chose ${ }^{36}$. Étant vide de tout contenu et «ne [comportant] en elle-même aucune diversité » ${ }^{37}$, c'est cette représentation d'un « sujet transcendantal » des pensées qui nous mène à concevoir le moi comme une «unité

argument qui aurait sa source chez Descartes, «It is much more illuminating (...) to view the argument as a more complete statement of the reasoning on which Leibniz (at least partly) based his rejection of materialism. (p. 509). Elle ne suggère pas cependant que Kant ait été influencé directement par le passage en question de la Monadologie ou par d'autres textes de Leibniz dans sa formulation du paralogisme.

32. A $352 ; 286$.

33. A $352-353 ; 286$.

34. A $353 ; 286$

35. A $355 ; 288$.

36. Ibid.

37. Ibid. 
absolue » et comme une chose entièrement simple. Ainsi s'explique l'erreur du psychologue rationnel quand celui-ci prend « la simplicité de la représentation d'un sujet » (ou d'un simple «quelque chose » porteur des pensées), pour « une connaissance de la simplicité du sujet lui-même » ${ }^{38}$.

La conséquence qui découle de la critique des deux paralogisnes est qu'il n'est pas plus possible de prouver «que tous les êtres pensants sont en soi des substances simples ${ }^{39}$ qu'il ne l'est de démontrer que ce sont des substances (et non des attributs d'autres substances). Le psychologue rationnel croit pouvoir « découvrir

38. Ibid. Karl AMERIKS, dans son livre sur les paralogismes (Kant's Theory of Mind. An Analysis of the Paralogisms of Pure Reason, Oxford : Clarendon Press, 1982) soutient (chapitre 2, intitulé «Immateriality ») que le moi, pour Kant, est simple en soi et que celui-ci ne rejette pas réellement le paralogisme de la simplicité ou, comme il le désigne, l' « argument de l'unité ». Il écrit : “(...) Kant himself fails to spell out the precise objection to the unity argument proper, and nothing he says in his discussion of simplicity elsewhere directly undercuts the conclusion of that argument. His major attack elsewhere on asserting the soul's simplicity typically stresses only that such an assertion couldn't prove the soul's permanence ( $\mathrm{A} 400-1$ ), and so it is really a criticism of the extended argument and not the unity argument.»(p. 63) Par l'«extented argument », Ameriks veut dire l'argument qui, à partir de sa simplicité, conclut à la permanence et à l'immortalité de l'âme (étant simple, elle serait indestructible) et, selon lui, c'est cet argument seulement qui soulève pour Kant des difficultés. Il défend cette interprétation en invoquant entre autres (si je le comprends bien), les deux raisons suivantes. (a) Kant, dans ses cours de métaphysique, aussi bien de la période critique que de la période pré-critique (comme en témoignant des notes prises par ses étudiants et publiées récemment dans les volumes XXVIII [1968, 1970 et 1972] et XXIX [1980 et 1983] des Gesammelte Schriften), présente l' " argument de l'unité " comme un argument entièrement concluant et il n'en fait pas la critique. (b) De plus, aucune des objections que fait Kant dans le chapitre des paralogismes contre l'argument n'est décisive et ne réussit à montrer en quoi il n'est pas convaincant. En ce qui concerne le point (a), il me semble qu'A meriks présuppose ici, ce qui est loin d'être évident, que les cours de métaphysique reflètent de façon exacte la pensée de Kant et sa position à l'égard des thèses et des arguments de la psychologie rationnelle. Kant, comme il le note, a une attitude moins critique vis-à-vis de ces thèses dans ses cours que dans le chapitre des paralogismes, mais cette différence pourrait s'expliquer peut-être par le fait qu'il s'agit de présenter dans ces cours un commentaire de la métaphysique de Baumgarten et non pas un exposé systématique de son propre point de vue (un de ces textes, publié dans le volume XXIX [1983] des Gesammelte Scbriften, porte le titre: "Bemerkungen ueber Metaphysic nach Baumgarten aus dem Vortrage des Herrn. Prof. Kant pro 1794/95 »). A propos du point (b), on peut remarquer que, même si les objections de Kant contre le paralogisme ne sont pas décisives, il reste qu'il a voulu faire cette critique de l'argument et qu'il le considérait par conséquent comme non concluant. Cependant, comme je le dirai dans la section 2, il y a peut-être effectivement une raison (différente de celle quavance Ameriks) de penser que pour Kant le moi est une chose simple.

39. B $409 ; 287$ 
des propriétés [du moi] qui n'appartiennent pas du tout à l'expérience possible (comme celle de la simplicité) [et savoir] d'un être pensant (...) quelque chose qui en concerne la nature ${ }^{40}$, mais il ne peut y avoir une connaissance qui dépasse les limites de toute expérience et qui prétend nous apprendre ce qu'est le sujet pensant en lui-même.

Il en découle également une conséquence pour la thèse que défend par ailleurs le matérialiste. Si le psychologue rationnel ne peut montrer que le moi est une substance simple en soi, le matérialiste, pour une même raison, ne peut pas non plus montrer que c'est une substance par essence composée plutôt que simple. La difficulté est la même pour les deux points de vue opposés, et le résultat de sa critique devrait être, dit Kant, de nous empêcher "d'une part de [nous] jeter dans le sein du matérialisme qui nie l'âme, d'autre part, de [nous] perdre avec extravagance dans un spiritualisme qui n'a aucun fondement pour nous dans la vie ${ }^{41}$. Il fait une remarque similaire à un autre endroit du chapitre dans un passage où il écrit ceci: "Ainsi donc une connaissance [du moi] que l'on cherche au-delà des limites de l'expérience possible (...) tant que nous la demandons à la philosophie spéculative [i.e. à la psychologie rationnelle], se résout en une espérance illusoire. Cependant, la sévérité dont fait preuve ici la critique, par le fait même qu'elle démontre l'impossibilité de décider dogmatiquement quelque chose touchant un objet (...) en dehors des limites de l'expérience, rend en même temps à la raison un service qui n'est pas sans importance (...) en l'assurant également contre toutes les assertions possibles du contraire ${ }^{42}$. En d'autres termes, il faut rester neutre sur le point de savoir laquelle des deux assertions est vraie, celle du psychologue rationnel et l'assertion contraire du matérialiste, dans la mesure où on sort inévitablement du domaine de l'expérience possiblé lorsqu'on essaie de déterminer si le moi, en tant que «chose en soi», devrait être considéré comme une substance simple et immatérielle ou bien comme une chose composée. De plus, cette critique « rend (...) à la raison un service qui n'est pas sans importance» puisqu'il demeure au moins

40. A $347=\mathrm{B} 405 ; 282$.

41. B $421 ; 306$.

42. B $423-424 ; 310-311$. 
possible que le moi soit une substance immatérielle et qu'ainsi on puisse croire (mais non pas savoir) que c'est une substance indestructible et immortelle (ou que c'est une âme au sens traditionnel du terme). En effet, la croyance dans l'immortalité de l'âme constitue un des "postulats » de la raison dans son usage " pratique », c'est-à-dire, comme le soutient Kant dans la seconde Critique, un des présupposés indispensables de la possibilité d'une loi morale. Si on peut déterminer les limites de notre connaissance du sujet pensant (des limites que dépasse autant le matérialiste que le psychologue rationnel), et montrer que le moi en soi est inconnaissable, alors on peut laisser ouverte de cette manière la possibilité d'une «croyance rationnelle» dans l'immortalité du moi.

Pour répondre à la question que j'ai posée au début, il semble donc qu'on puisse décrire comme suit la position de Kant. Le moi pensant pourrait être aussi bien une substance immatérielle qu'une chose matérielle: il n'y a pas davantage de contradiction à dire qu'une pensée, conçue comme la réunion de plusieurs représentations, est inhérente à une substance composée qu'il y en a à soutenir le point de vue contraire. Étant donné quaucune expérience ne peut nous dire quoi que ce soit sur le sujet tel qu'il est en luimême, le matérialiste ne peut prétendre savoir mieux que le psychologue rationnel ce qui appartient à l'essence du moi. Il se pourrait que celui-ci soit effectivement une substance simple et capable d'une existence indépendante de la matière (quoiqu'il se pourrait aussi que le contraire soit vrai) et, par conséquent, rien ne s'oppose à une croyance dans son immortalité.

Mais Kant s'en tient-il à cette position de «neutralité » ̀̀ l'égard des deux doctrines? Comme je l'ai noté au début, on trouve dans le texte du chapitre des paralogismes un argument contre le matérialisme, lequel vise à montrer que, même si on ne peut rien dire sur la nature du sujet tel qu'il est en lui-même, nous pouvons au moins savoir qu'il n'est pas en soi une chose divisible et de même essence que la matière. Je vais exposer dans ce qui suit cet argument.

Un passage dans lequel Kant présente sa raison de rejeter le matérialisme se lit comme suit: 
Quel besoin pouvons-nous avoir d'une psychologie [rationnelle]? Nous la voulons surtout sans doute en vue de mettre notre moi pensant à l'abri du danger du matérialisme. Mais à cela suffit le concept rationnel que nous avons donné de notre moi pensant. Tant s'en faut, en effet, qu'avec ce concept il reste la moindre crainte de voir s'évanouir, si l'on supprime la matière, toute pensée et l'existence même des êtres pensants : au contraire il est clairement démontré que, si je supprime le sujet pensant, il faut que tout le monde corporel s'évanouisse, comme n'étant rien que le phénomène dans la sensibilité de notre sujet et un mode de représentation du sujet ${ }^{43}$.

Kant semble dire dans ce passage qu'il partage le but du psychologue rationnel, à savoir montrer qu'il y a une différence de nature entre le moi pensant et la matière, mais, en même temps, que le «concept rationnel» (Vernunftbegriff) qu'il a donné d'un sujet pensant devrait suffire à mettre le moi «à l'abri du danger du matérialisme ». Il n'explique pas ce qu'il entend, plus exactement, par ce «concept rationnel » du moi, mais l'affirmation est plutôt surprenante étant donnée la critique des paralogismes, laquelle implique au contraire l'impossibilité de savoir si le moi est ou bien n'est pas, en soi, une substance immatérielle. En quoi consiste donc cet argument?

L'argument tire parti d'une thèse centrale de Kant introduite au début de la Critique de la raison pure, dans l'Esthétique transcendantale, la thèse de l' « idéalité » (ou subjectivité) de l'espace et des phénomènes donnés dans l'espace. D'après cette thèse, à laquelle j'ai fait allusion plus haut, l'espace doit être conçu comme quelque chose qui se trouve «en nous»: il est un mode de représentation des objets extérieurs propres aux êtres humains et il n'existe pas objectivement comme une chose en soi indépendante de la faculté qu'a le sujet de se représenter les objets (faculté que Kant appelle «sensibilité »). On lit entre autres, dans l'Esthétique, le passage suivant: « Nous ne pouvons parler de l'espace, de l'être étendu, etc., qu'au point de vue de l'homme. Si nous sortons de la condition subjective sans laquelle nous ne saurions recevoir d'intuitions extérieures, c'est-à-dire être affectés par les objets, la représentation de l'espace ne signifie plus rien. Ce prédicat n'est joint aux choses qu'en tant qu'elles nous apparaissent, c'est-à-dire qu'elles sont des objets de la sensibilité (...) [Et] nous ne saurions

43. A $383: 309$. 
faire des conditions particulières de la sensibilité les conditions de possibilité des choses, mais celles seulement de leur manifestation phénoménale (...) ${ }^{44}$. Comme forme a priori, l'espace est la structure nécessaire à travers laquelle nous apparaissent les objets ; il n'existe pas en soi en dehors du sujet et les objets représentés en lui ne possèdent pas en soi des propriétés telles qu'avoir une forme ou avoir des dimensions : ce n'est que leur manifestation phénoménale, la façon dont ils apparaissent au sujet, qui est soumise aux conditions subjectives de la sensibilité. Or, être une chose étendue et être matériel, selon Kant, sont des propriétés coextensives. Pour lui comme pour Descartes, c'est une seule et même réalité que l'on désigne quand on parle d'une substance étendue, d'une substance matérielle ou bien d'une chose composée et divisible. Mais si être dans l'espace n'est pas une propriété constitutive des choses mais seulement une propriété liée à leur manifestation phénoménale, alors on peut en dire autant de la propriété être une chose matérielle. Comme il l'écrit dans ce passage, "le monde corporel (...) [n'est] rien que le phénomène dans la sensibilité de notre sujet et un mode de représentation du sujet». Pour le matérialiste, «si l'on supprime la matière », on abolit également «toute pensée et l'existence même des êtres pensants». Pour Kant, c'est l'inverse qui est vrai: si on supprimait les êtres pensants, la matière disparaîtrait avec eux dans la mesure où les choses matérielles, ce qui se trouve représenté dans l'intuition $a$ priori de l'espace, n'existe que du point de vue des sujets bumains. Il suit de là que si la matérialité et l'étendue sont uniquement des modes de représentation inséparables de la façon propre aux êtres humains de percevoir les objets extérieurs, alors le sujet pensant n'est pas lui-même une chose matérielle ou étendue. En d'autres termes, puisque être matériel est une propriété relative au sujet et à sa faculté de représentation et non une propriété absolue que posséderaient en soi les objets, le sujet, pour qui les choses apparaissent comme juxtaposées dans l'espace, ne peut être luimême une substance étendue et matérielle.

Dans l'Esthétique, Kant soutient que nous connaissons les objets seulement tels qu'ils nous apparaissent, conformément aux conditions subjectives de la sensibilité. À d'autres endroits, comme

44. A $26-27$ B $42-43 ; 58-59$ 
dans le chapitre des paralogismes et, en particulier, dans la critique du «paralogisme de l'idéalité du rapport extérieur », il affirme que ce ne sont pas réellement des objets que nous connaissons empiriquement, mais des phénomènes, lesquels ne sont que des représentations provoquées dans l'esprit par les objets, ces objets qu'il qualifie d'extérieurs «au sens transcendantal» ${ }^{45}$. Or on peut dire la même chose selon lui de la matière: ce n'est pas l'objet transcendantal ou les objets "extérieurs en soi» ${ }^{46}$ "que nous entendons en parlant des représentations de la matière et des choses corporelles; car celles-ci ne sont que des phénomènes, c'est-à-dire que de simples modes de représentations qui ne se trouvent jamais qu'en nous (...) ${ }^{47}$. La matière dit-il encore, n'est "qu'une espèce de représentations (...) qu'on appelle extérieures, non parce qu'elles se réfèrent à des objets extérieurs en soi, mais parce qu'elles rapportent les perceptions à l'espace (...), tandis que l'espace lui-même est en nous " ${ }^{48}$. Au philosophe (qu'il appelle l'«idéaliste sceptique ») qui trouve douteuse la réalité de la matière parce qu'il estime qu'elle est conclue seulement comme une des causes possibles des représentations et que, pour cette raison, son existence ne peut jamais être tenue pour certaine, Kant répond que «la matière [possède] une réalité qui n'a pas besoin d'être conclue, mais qui est immédiatement perçue ${ }^{49}$, dans la mesure où «ces choses extérieures, à savoir la matière avec toutes ses formes (...), ne sont que de simples phénomènes, c'est-à-dire que des représentations en nous, de la réalité desquelles nous avons conscience immédiatement ${ }^{50}$. Kant, en ce sens, pourrait formuler également comme suit son argument: la matière n'est qu'un certain type de représentation, ces représentations données par le sens externe et dont la forme a priori est l'espace, et, en tant que représentation, la matière n'existe que dans et pour le sujet, de sorte que celui-ci ne peut pas être lui-même quelque chose d'étendu et de matériel.

Qu'est-ce qu'on peut conclure de cet argument en ce qui concerne la position de Kant à l'égard du dualisme cartésien et à

45. A $372 ; 302$.

46. A $370 ; 300$.

47. A $372 ; 302$

48. A $370 ; 300$.

49. A $371 ; 301$.

50. Ibid. 
l'égard du matérialisme? Il semble qu'on puisse en déduire non seulement que le sujet pensant est immatériel, mais aussi, assez curieusement, qu'il est une substance simple. On arrive à cette conclusion de la façon suivante. D'une part, les choses apparaissent comme données dans l'espace, mais ce n'est que du point de vue du sujet que l'on peut leur attribuer cette propriété. De plus, dans la forme a priori de l'espace, les choses sont représentées comme divisibles et composées, parce que dans l'espace il n'existe rien de simple: «Il n'y a pas dans l'espace de réel qui soit simple, car les points (...) ne sont que des limites mais non quelque chose qui serve comme partie [simple], à constituer l'espace " ${ }^{51}$. D'autre part, ce qui est vrai de l'étendue l'est également du caractère composé des objets. Comme on peut le lire dans le commentaire du paralogisme de la simplicité, être étendu (ausgedebnt) et être composé (zusammengesetzt) sont «[des] prédicats [qui] ne concernent que la sensibilité et son intuition, en tant que nous sommes affectés par des objets ${ }^{52}$, donc des prédicats qui appartiennent non aux choses telles qu'elles sont en soi mais des prédicats qui dépendent essentiellement de la façon propre au sujet de se représenter les objets. Si être composé est une propriété des objets seulement tels qu'ils apparaissent du point de vue du sujet, une propriété liée à leur manifestation phénoménale, il s'ensuit que le sujet lui-même n'est pas composé et, par conséquent, puisque quelque chose est soit simple, soit composé, il s'ensuit qu'il est une substance simple. Par cet argument contre le matérialisme, Kant revient, semble-t-il, à la thèse du psychologue rationnel pour qui le moi est une chose non divisible et immatérielle.

En ce point, deux questions se posent. (1) Si, comme je l'ai dit plus haut, il n'est pas possible de savoir quoi que ce soit sur l'essence du « sujet transcendantal » des pensées, alors, apparemment, on ne peut pas non plus maintenir que celui-ci est simple par nature. Est-ce qu'affirmer la simplicité de nature du sujet pensant ne présente pas en ce sens une difficulté évidente pour Kant? (2) D'autre part, à supposer que le moi soit effectivement une substance simple, comme est censé le montrer l'argument, est-ce que l'on doit nécessairement en déduire qu'il y a selon Kant une différence de nature entre le moi et les choses matérielles et que sa position,

51. B $419 ; 304$.

52. A $358 ; 290$. 
en définitive, ne diffère en aucune façon de celle que défend le psychologue rationnel? Je commence par la seconde de ces deux questions.

Bien qu'à première vue ceci puisse sembler paradoxal, l'argument contre le matérialisme n'implique pas du tout qu'il y a une différence de nature entre le moi et la matière ou, comme le soutient le psychologue rationnel, qu'il existe, d'un côté, des choses simples et immatérielles et, de l'autre, des substances divisibles et matérielles. L'argument disait ceci: le moi est une substance indivisible et immatérielle parce que la matérialité et la divisibilité sont des propriétés uniquement des objets tels qu'ils apparaissent du point de vue du sujet. Or, il est évident que ce que l'on dit du sujet pensant, il est permis aussi de le dire des objets eux-mêmes qui lui apparaissent à travers leur manifestation phénoménale : ces objets ne sont pas non plus en soi dans l'espace, ils n'ont pas une forme ou des dimensions, ce ne sont pas des choses que l'on pourrait qualifier de matérielles ou de composées dans la mesure où, toutes ces propriétés, elles ne les possèdent que relativement à la constitution particulière de la sensibilité humaine : l'objet transcendantal, dit Kant, ou l'objet «extérieur en soi », est un "quelque chose [qui] n'est ni étendu (...), ni composé, puisque tous ces prédicats ne concernent que la sensibilité et son intuition (...). Ces expressions [ «être étendu », «être composé »] ne nous font pas du tout connaître ce qu'est l'objet lui-même; elles nous montrent seulement que ces prédicats des phénomènes extérieurs ne peuvent pas être attribués à cet objet considéré comme tel, c'est-à-dire en lui-même et sans aucun rapport avec les sens extérieurs" ${ }^{53}$. D'après cela, on pourrait reformuler l'argument contre le matérialisme comme suit: si la matérialité et la divisibilité sont des propriétés inhérentes seulement aux phénomènes et non aux objets tels qu'ils sont en eux-mêmes, alors il n'existe rien en soi de matériel et de divisible et, en particulier, le sujet pensant n'est pas lui-même une substance matérielle et divisible. En ce sens, l'argument revient à soutenir qu'il n'existe aucune chose à l'essence de laquelle appartiennent ces propriétés. Contre le matérialiste, Kant soutient qu'aucune substance, en soi ou par essence, n'est une substance matérielle. Et ceci signifie, apparemment, que toute chose, en soi,

53. A $358-359 ; 290$. 
est simple et immatérielle et, par conséquent, qu'il n'y a aucune différence de nature entre le sujet pensant et les objets extérieurs en soi, du moins pour ce qui concerne leur caractère simple ou composé. Donc, même si on affirme avec le psychologue rationnel la simplicité de nature du sujet en soi, il ne s'ensuit pas pour autant qu'il existe, d'une part, des substances simples et immatérielles et, de l'autre, des substances divisibles et matérielles. Au contraire, toute chose, par essence, serait selon Kant non divisible et immatérielle.

La position qui vient d'être décrite est évidemment très différente de celle que j'ai attribuée plus haut à Kant dans la section 1. Dans sa critique des paralogismes de la substantialité et de la simplicité, Kant soutient qu'on ne peut connaître la nature du sujet pensant en lui-même, de sorte qu'il est impossible de déterminer si le moi est une substance simple ou composée et de décider à qui donner raison, au psychologue rationnel ou bien au matérialiste. L'argument contre le matérialisme vise à montrer plutôt qu'il n'existe rien par essence de composé et de matériel, ni l' «objet» du sens interne (ou le sujet transcendantal des pensées), ni les objets du sens externe (ou les « choses en soi »). Mais, pour revenir à la question (1), il y a ici une difficulté : affirmer la simplicité et l'immatérialité du sujet pensant et des objets extérieurs contredit, semble-t-il, l'idée, absolument fondamentale pour Kant, d'après laquelle on ne peut connaître la nature des choses en soi.

Il faut faire une distinction, je pense, entre la question de l'immatérialité du moi pensant ou, en général, des choses en soi, et la question de leur simplicité. Lorsque Kant affirme la nonmatérialité des choses en elles-mêmes, le fait qu'elles ne soient pas données dans l'espace, il ne les caractérise pas de façon positive, il nous dit seulement ce qui n'appartient pas à leur essence. De ce que le moi est une chose immatérielle, par exemple, on ne peut rien déduire en ce qui concerne sa nature; il ne découle pas, en particulier, qu'il serait en soi une chose pensante, car il existe peutêtre d'autres possibilités que nous ne sommes pas en mesure de nous représenter parce que (dirait-il) elles excèdent les limites de notre entendement ou les capacités de l'imagination humaine (à supposer, bien sûr, que la pensée exclut effectivement la matérialité). Mais la situation est différente si on dit que l'objet (ou le sujet) transcendantal est une substance non composée : il ne reste dans ce 
cas qu'une seule autre possibilité, à savoir que cette substance est par nature simple. Pour prendre un autre exemple qui illustre cette différence, si je dis d'une chose qu'elle est non bleue, je communique peu d'information sur ses propriétés parce qu'il se pourrait qu'elle soit verte, jaune, ou bien rouge, etc. Mais si d'une pièce de métal je dis qu'elle est non malléable, on peut en déduire immédiatement qu'elle est cassante, parce qu'il n'existe que deux possibilités : être malléable ou bien être cassant. De façon analogue, il semble qu'attribuer la propriété être immatériel à une chose communique moins d'information à son sujet que si on lui attribue la propriété non composé : elle pourrait être immatérielle au sens où un esprit ou Dieu est immatériel, ou bien (peut-être) au sens où le nombre deux est immatériel; et il y a sans doute encore d'autres possibilités. Mais être non composé, au sens de être dénué de parties, implique nécessairement être simple ou non divisible. Bref, s'il est possible de savoir à propos des choses en soi qu'il s'agit de substances par nature simples, comme semble le soutenir Kant, alors on affirme à leur sujet quelque chose de beaucoup plus précis que si on dit uniquement que ce sont des substances par essence immatérielles. Il y aurait donc contradiction entre la critique du paralogisme de la simplicité, d'après laquelle on ne peut prouver la simplicité en soi de l'âme, parce qu'on ne peut rien savoir en général sur la nature des choses en soi, et l'argument contre le matérialisme qui, apparemment, entraîne la conséquence contraire.

Kant, cependant, pourrait répondre à cela qu'il n'existe en réalité aucune différence entre le prédicat immatériel et le prédicat non composé. En effet, attribuer la non-matérialité et la noncomposition aux choses en soi, c'est, dans les deux cas, les caractériser uniquement de façon négative sans dire quoi que ce soit, positivement, sur les propriétés qui constituent leur essence. Il pourrait soutenir qu'il existe (ou pourrait exister) d'autres possibilités que la seule alternative simple/composé et que, peut-être, les choses en soi ne sont ni simples, ni composées, mais qu'elles possèdent des propriétés entièrement inconnues de nous et n'ayant aucune espèce de similitude avec les propriétés empiriques familières que l'on rencontre dans les phénomènes. Toutefois, il semble que selon lui il réste au moins possible que toute chose soit en ellemême simple, alors qu'il ne se peut pas que quelque chose soit composé et matériel. 
Retenons donc de l'argument contre le matérialisme qu'il démontre seulement l'immatérialité des choses en soi et non pas leur simplicité, et tirons les conséquences en ce qui concerne la position de Kant vis-à-vis du dualisme et du matérialisme. D'après la critique des paralogismes que j'ai exposée dans la section 1, la difficulté dans le point de vue du dualiste est la même que pour le point de vue du matérialiste: si on ne peut rien connaître de la nature des choses en soi, nous ne sommes pas en mesure de savoir lequel des deux points de vue est vrai (quoique peut-être, semblait dire Kant, l'un des deux est vrai). Kant, maintenant, fait une critique différente et sa critique, il me semble, entraîne le rejet des deux points de vue opposés. Le dualiste et le matérialiste partagent en effet le présupposé d'après lequel la matière est une chose en soi ou, autrement dit, qu'il y a quelque chose dont l'essence se définit par la matérialité, l'étendue et la composition, et c'est à ce présupposé commun que s'attaque Kant par son argument. Le matérialiste soutient (a) qu'il y a identité de nature entre le moi pensant et la matière et $(b)$ que l'essence de la matière est d'être une substance étendue et divisible. À cela, donc, Kant répond, premièrement, que rien n'est en soi composé et étendu mais aussi, deuxièmement, qu'il pourrait effectivement y avoir identité de nature, non pas cependant entre le moi et la matière, mais entre le moi et le «substrat » de la matière, c'est-à-dire ce quelque chose qui, dans son phénomène, nous apparaît comme matériel mais ne l'est pas en soi. Il pourrait y avoir identité de nature parce que ni le moi en soi, ni le substrat des phénomènes extérieurs n'est intrinsèquement quelque chose de matériel ${ }^{54}$; en particulier, il se pourrait que le sujet et l'objet trancendantal soient tous deux des substances indivisibles. Le psychologue rationnel, de son côté, soutient $(a)$ que le moi est un être pensant en lui-même, $(b)$ qu'il existe d'autres substances différentes par essence du moi pensant, et $(c)$ que ces substances sont par nature matérielles et composées. Pour Kant, i1 se pourrait que $(a)$ et $(b)$ soient vrais, mais, à nouveau, $(c)$ est faux :

54. KANT écrit ceci : « la matière (...) n'est autre chose qu'une simple forme ou un certain mode de représentation d'un objet inconnu formé par l'intuition qu'on nomme le sens extérieur.(...) La marière ne signifie donc pas une espèce de substance si complètement différente et si totalement hétérogène de l'objet du sens interne (de l'âme) (...) 》 (A $385 ; 311$ ). Si la matière, autrement dit, n'existe pas en soi, alors l'objet transcendantal du sens externe n'est pas en lui-même matériel et, par suite, il se pourrait qu'il soit de même nature que l'objet (en soi) du sens interne. 
il est possible que le moi soit une substance pensante (que la pensée appartienne à sa nature), qu'il y ait d'autres substances que des substances pensantes, mais il n'est pas possible que celles-ci soient par essence matérielles. En somme, pour lui, on peut envisager les deux possibilités suivantes: soit une forme de dualisme (non cartésien) entre le moi et d'autres substances, lesquelles substances seraient non pensantes (différentes du moi dans leur essence) quoique immatérielles; soit une forme de monisme d'après lequel il n'existe qu'un seul type de substance, et celle-ci, également, serait immatérielle.

D'après ce qui précède, Kant paraît moins critique à l'endroit de la thèse du psychologue rationnel qu'il ne l'est à l'endroit de celle du matérialiste. Cette forme de dualisme entre le moi pensant et les autres substances (dont on ne connaît pas la nature) demeure après tout très proche du dualisme cartésien de la substance pensante et de la substance matérielle, la seule différence étant évidemment que, pour Descartes, il existe de ces substances dont l'«attribut principal consiste dans l'étendue. Mais on ne peut pas en dire autant du monisme et du matérialisme: le point de vue d'après lequel il n'existe qu'un seul type de substance est passablement éloigné du matérialisme dans la mesure où le monisme admet comme variante, entre autres, la doctrine à laquelle (dans un passage que je cite plus loin) Kant donne le nom de " pneumatisme », c'est-à-dire la doctrine qui revient à soutenir que toute substance, par essence, est une substance pensante; et on pourrait difficilement imaginer deux points de vue aussi différents l'un de l'autre que le matérialisme, qui consiste à soutenir que tout est matière, et le pneumatisme, pour lequel il n'existe que des êtres pensants. Ces deux points de vue, pourtant, s'opposent au dualisme dans la mesure où ils affirment tous deux l'existence d'un seul type de substance.

Cependant, il existe d'autres possibilités. Jusqu'ici, on a supposé, en accord avec le point de vue du psychologue rationnel, qu'une personne (un individu humain) pouvait être formée de deux entités différentes et jointes ensemble : une substance pensante en soi (ou un esprit) et une autre substance qui, par les sens externes, apparaît sous l'aspect d'un corps bien qu'elle ne soit pas en ellemême corporelle. Rien du moins n'excluait cette possibilité. Dans un passage du commentaire du paralogisme de la simplicité, on 
trouve mentionnée une autre hypothèse: on peut concevoir, comme le dualiste cartésien, qu'une personne soit constituée par la réunion de deux substances distinctes, mais il est possible également qu'il n'y ait dans une personne qu'une seule substance, laquelle serait en elle-même pensante, mais nous apparaîtrait en même temps, dans son phénomène, comme une chose matérielle.

Le passage en question débute par un rappel de la conclusion de l'argument contre le matérialisme: "Nous avons établi d'une manière incontestable, dans l'Esthétique transcendantale, que les corps sont de simples phénomènes de notre sens externe et non des choses en soi. D'après cela, nous pouvons dire avec raison que notre sujet pensant n'est pas corporel (...) ${ }^{55}$. Puisque le moi n'est pas en lui-même une substance corporelle, alors on peut supposer, entre autres, qu'il est en soi une chose pensante et aussi, comme pour Descartes, une substance indivisible, donc quelque chose en soi dénué de parties et qui a des représentations, des intentions, des sentiments, etc. Mais cette supposition n'est pas incompatible avec l'hypothèse d'après laquelle une personne serait formée d'une seule substance. Kant écrit ceci : la substance « qui sert de fondement aux phénomènes extérieurs et qui affecte notre sens de telle sorte qu'il reçoit les représentations d'espace, de matière, de figure, etc., ce quelque chose, à titre de noumène (ou, plutôt, comme objet transcendantal), pourrait en même temps être [une chose qui pense] quoique, par la manière dont notre sens extérieur en est affecté, nous n'ayons pas d'intuition de représentations, de volitions, etc., mais simplement des intuitions de l'espace et de ses déterminations. " 56 En effet, dit-il, "les prédicats du sens intérieur, représentations et pensées, ne lui sont pas contradictoires $\$$ 57: l'objet transcendantal pourrait être une chose pensante bien qu'il nous apparaisse plutôt, par le sens externe, comme une chose dont les propriétés sont l'étendue et la composition. Il n'y a aucune contradiction à attribuer toutes ces propriétés simultanément à une seule et même substance parce que la pensée est attribuée à l'objet lui-même, tandis que les propriétés telles que la composition et l'étendue se rapportent à son seul phénomène. En outre, cette substance, qui nous apparaît comme composée et matérielle,

55. A $357 ; 289$.

56. A $358 ; 290$.

57. Ibid. 
pourrait très bien être en elle-même une chose simple: «[La matière] est simplement un phénomène extérieur dont le substratum n'est connu par aucun prédicat que l'on puisse indiquer; par suite, je puis bien admettre de ce substratum qu'il est simple en soi, bien que suivant la manière dont il affecte nos sens, il produise en nous l'intuition de l'étendu et, par suite, du composé (...) ${ }^{58}$. Une personne pourrait donc n'être constituée que d'une seule substance, s'apparaissant à elle-même par son sens interne comme une chose qui a des pensées, des sentiments, des volitions, etc., et apparaissant à autrui par le sens externe (ou à elle-même si elle observe son propre corps) comme une substance étendue et composée. «De cette manière », dit Kant, « la même chose qui, sous un rapport [en tant que phénomène], serait appelée corporelle, serait en même temps, sous un autre rapport [comme chose en soi], un être pensant (...). Ainsi tomberait l'expression que les âmes seules (comme espèces particulières de substances) pensent; il serait mieux de dire, comme on le fait habituellement, que les hommes pensent, c'est-à-dire que la même chose qui est étendue, en tant que phénomène extérieur, est intérieurement (en elle-même) un sujet non composé, mais simple, et qui pense ${ }^{59}$. D'après ce passage, non seulement il pourrait y avoir identité de nature entre le moi en soi et le substrat de la matière (en ce sens qu'ils pourraient être tous deux des substances pensantes indivisibles), mais il se pourrait aussi qu'une personne, un être possédant à la fois des propriétés physiques et des propriétés non physiques, ne soit constituée en réalité que d'une seule substance, une chose à laquelle on pourrait attribuer toutes ces propriétés, les unes (les propriétés non physiques) étant des caractéristiques de la chose elle-même (l'être pensant), les autres (les propriétés physiques) des qualités de la chose telle qu'elle nous apparaît (en tant qu'objet matériel).

Lorsque Kant dit vouloir mettre le moi pensant «à l'abri du danger du matérialisme », on peut voir maintenant que ce n'est pas au sens où il s'agirait de montrer qu'il y a dans l'univers deux genres de substances d'essences opposées et incompatibles, des substances pensantes simples et des substances matérielles ou divisibles. Il n'affirme pas, dans le passage que je viens de citer du

58. A 359;291.

59. A $359-360 ; 290$. 
commentaire du paralogisme de la simplicité, que le moi est une chose simple; il fait l'hypothèse qu'il est simple et il se pose ensuite la question de savoir s'il est possible d'en déduire quelque chose "par rapport à l'hétérogénéité ou à l'homogénéité de l'âme avec la matière ${ }^{60}$. La réponse à cette question est négative, selon lui, parce qu'il se pourrait que le moi soit une substance indivisible sans qu'il y ait pour autant une différence de nature entre cette chose et la matière (ou, plus exactement, entre le moi en soi et le substrat de la matière). Il est même possible, dit-il, que se rencontrent dans une personne non pas deux substances d'essences différentes, comme le croit le dualiste, mais une seule, une substance se révélant à elle-même par le sens interne comme ayant des représentations et des pensées, et par le sens externe comme ayant une forme, des dimensions et comme entretenant des relations spatiales avec d'autres substances. On peut cependant rejeter le matérialisme, selon lui, c'est-à-dire montrer que le sujet pensant n'est pas composé et matériel, et ainsi admettre au moins la possibilité qu'il soit une substance par nature indivisible en autant que cela n'implique pas le dualisme classique de la substance pensante et de la substance (par essence) étendue.

Pour revenir brièvement à la question de l'immortalité, il semble possible également que, en tant que simple, le moi soit une substance indestructible: étant dépourvu de parties, il ne se pourrait pas que le moi cesse d'exister par décomposition ou division. Toutefois, on trouve dans la seconde édition de la première Critique un passage important où il soutient que, même en supposant sa simplicité, il ne suit pas nécessairement que le moi est indestructible. C'est ce qu'on peut voir, selon lui, si on fait une distinction (qu'il a introduite dans l'Analytique des principes) entre deux types de grandeurs, les «grandeurs extensives» et les "grandeurs intensives ». Il écrit ceci : « même en accordant à l'âme cette nature simple, puisque (...) elle ne renferme pas de parties placées les unes en dehors des autres, ni, par suite, de grandeur extensive, on ne saurait pourtant lui refuser (...) une grandeur intensive, c'est-à-dire un degré de réalité relativement à toutes ses facultés (...), [et] ce degré peut décroître de plus en plus indéfiniment [de sorte que] la prétendue substance (...) peut se réduire à rien,

60. A $357 ; 289$. 
sinon par décomposition, du moins par une déperdition graduelle de ses forces (remissio) ou par consomption (...) ». En effet, dit-il, "la conscience même a toujours un degré qui peut toujours diminuer; il en est de même, par conséquent, du pouvoir d'avoir conscience de soi et de tous les autres pouvoirs [de l'esprit] ${ }^{61}$. Kant ne tire pas cependant dans ce passage la conséquence que l'âme est une substance destructible. Apparemment, selon lui, tout ce que l'on peut conclure de cette remarque c'est que « la permanence de l'âme », donc son immortalité, «n'est pas démontrée et même [qu'elle] n'est pas démontrable ${ }^{62}$. Si on voulait montrer que l'âme est destructible et qu'elle peut cesser d'exister, il faudrait en effet que nous sachions qu'elle est simple (mais ce n'est pas quelque chose que nous pouvons savoir) et de plus, à supposer qu'elle soit simple, qu'elle possède une grandeur intensive; cependant il n' indique pas dans ce passage si toute chose, y compris une chose en soi, doit nécessairement avoir l'un ou l'autre de ces deux types de grandeurs.

Les objections que fait Kant à l'adresse des thèses de la psychologie rationnelle, comme je l'ai remarqué, restent nuancées, contrairement à sa critique du matérialisme : il n'est pas question dans ce chapitre de montrer que l'âme n'est pas une substance simple et indestructible; il s'agit uniquement de montrer qu'on ne peut connaître ses propriétés. De même, comme on l'a vu, il se pourrait que le moi soit un être pensant en soi, bien que, même si on fait cette supposition, on ne puisse er déduire qu'il y a une différence de nature entre les êtres pensants et la matière. Pourtant, on trouve dans le texte des paralogismes au moins une raison de croire que pour Kant il n'existe pas de substances par nature pensantes. Dans la section 1, j'ai noté que pour Kant une personne se connaît elle-même, par le sens interne, non telle qu'elle est en soi mais seulement telle qu'elle s'apparaît comme phénomène, et j'ai soulevé la question de savoir de quelle façon ou sous quel aspect, par ce phénomène, quelqu'un s'apparaît à lui-même. Il semble que pour Kant les phénomènes du sens interne soient, entre autres, des représentations, des sensations, des sentiments, des intentions, et l'ensemble des contenus de conscience d'une

61. B 414; 295-296.

62. Ibid. 
personne, c'est-à-dire ce que l'on peut désigner par le terme générique de "pensées». Le moi s'apparaîtrait alors, par ces phénomènes, comme une chose qui a des représentations, des sentiments, etc. ou comme une chose dont la seule propriété est la "pensée » au sens que Descartes donnait à ce mot. Or, si le moi s'apparaît comme quelque chose qui a des pensées et si la pensée appartient seulement à son phénomène, alors il ne possède pas en lui-même cette propriété et on ne peut plus dire, contrairement au point de vue du psychologue rationnel, qu'il se pourrait que la pensée appartienne à son essence. L'argument contre le matérialisme s'appuie sur l'idée que, bien que les objets extérieurs nous apparaissent comme ayant des propriétés spatiales, ils ne les possèdent pas en eux-mêmes. Kant pourrait employer un argument analogue dans le but de montrer que le moi n'est pas en lui-même un être pensant : on pourrait dire, certes, que le moi s'apparaît par le sens interne comme un être pensant, mais non pas qu'il possède en luimême cette propriété. La conséquence, contrairement à ce que l'on a supposé jusqu'ici, serait qu'il n'existe pas davantage de substances pensantes en elles-mêmes qu'il n'existe de choses en soi matérielles. Est-ce que Kant a lui-même tiré cette conséquence ? Il y a dans le chapitre des paralogismes un passage, mais un seul à ma connaissance, où il fait précisément cette objection à la doctrine de la psychologie rationnelle. Le passage est celui-ci :

[Si] l'on voulait étendre, comme il arrive ordinairement, le concept du dualisme et le prendre dans le sens transcendantal, alors ni ce concept, ni le pneumatisme qui lui est opposé d'une part, ou le matérialisme de l'autre, n'aurait plus le moindre fondement, puisque l'on fausserait alors la détermination de ses concepts et que l'on prendrait la différence du mode de représentation des objets, qui nous demeurent inconnus dans ce qu'ils sont en soi, pour une différence de ces choses mêmes. Le moi, représenté dans le temps par le sens intérieur, et les objets représentés dans l'espace hors de moi, sont, à la vérité, des phénomènes spécifiquement tout à fait distincts, mais ils ne sont pas conçus pour cela comme des choses différentes. L'objet transcendantal, qui sert de fondement (zum Grunde liegt) aux phénomènes extérieurs, tout comme celui qui sert de fondement à l'intuition interne, n'est ni matière, ni un être pensant en lui-même (weder Materie, nocb ein denkend Wesen an sich selbst), mais un principe (Grund) à nous inconnu des phénomènes qui nous fournissent le concept empirique de la première espèce [le concept d'un objet extérieur] aussi bien que de la seconde espèce [le concept d'un être pensant]. ${ }^{63}$

63. A 379-380; 307, trad. mod. 
En quoi consiste cette différence entre les deux types de phénomènes dont il est dit qu'ils sont «spécifiquement tout à fait distincts ». Nous savons que les phénomènes du sens externe sont donnés dans l'espace, et que l'objet qui leur correspond, le substrat des phénomènes, nous apparaît comme ayant une forme et des dimensions; alors que les phénomènes du sens interne nous apparaissent comme des réalités qui sont données non dans l'espace mais seulement dans le temps : une pensée ne possède ni forme, ni dimensions et ses seules relations avec d'autres pensées sont des relations temporelles. Mais on pourrait être tenté de prendre « [cette] différence du mode de représentation des ubjets (...) pour une différence de ces choses mêmes ». D'une part, si les pensées ne possèdent aucune des propriétés des choses matérielles et étendues, on pourrait croire qu'elles se rapportent à quelque chose d'immatériel et d'inétendu, quelque chose dont la seule propriété est d'être le sujet des pensées, donc à une chose par nature pensante. D'autre part, les phénomènes du sens externe étant représentés comme des objets placés dans l'espace, on pourrait croire que l'espace existe en lui-même et que les objets extérieurs possèdent réellement des propriétés comme avoir une forme ou avoir des relations spatiales avec d'autres objets. Il y aurait, d'un côté, des substances pensantes et immatérielles (ou hors de l'espace) et, de l'autre, des choses en elles-mêmes matérielles et placées dans l'espace. Mais, en réalité, dit Kant, bien que les objets du sens externe et l'objet du sens interne soient représentés, dans leur phénomène, comme des choses différentes, ils pourraient ne pas être en eux-mêmes différents, car les objets du sens externe ne sont pas en eux-mêmes matériels et étendus et, comme il le dit ici, l'objet du sens interne n'est pas en soi un être pensant. La différence des modes de représentation n'implique pas une différence dans la nature des objets, et il est même possible qu'il s'agisse d'un seul et même objet nous apparaissant extérieurement comme une chose ayant des propriétés spatiales et intérieurement (par le sens interne) comme un être pensant. Kant en déduit qu'il faut rejeter non seulement le matérialisme, mais également le pneumatisme et le dualisme, si on prend ces trois doctrines au « sens transcendantal ", c'est-à-dire comme des assertions sur le moi et la matière en soi. On doit rejeter le matérialisme parce que rien n'est en soi matériel, le pneumatisme parce qu'il n'existe pas d'êtres pensants en soi et, pour les mêmes raisons, le dualisme, qui affirme la réalité 
en soi à la fois de la substance matérielle et de la substance pensante.

Plus haut, j'ai dit qu'il est (au moins) possible pour Kant qu'il existe des êtres dont l'essence réside dans la pensée, alors que dans le texte qui vient d'être cité il maintient le contraire. Même s'il s'agit du seul endroit dans le chapitre où c'est ce qu'il soutient, il semble que le passage soit suffisamment explicite pour qu'on puisse y avoir une expression fidèle de son point de vue. Ce qui nous mène évidemment à poser la question de la cohérence d'ensemble de sa critique de la psychologie rationnelle. Est-ce que les positions successives que $j$ 'ai décrites ne sont pas, en définitive, incompatibles entre elles?

Il me semble qu'on peut se représenter ses objections en les situant à plusieurs niveaux, chacune se rapportant à une hypothèse différente, et dans une telle façon de procéder il n'y a aucune contradiction. D'après une première hypothèse, qui correspond à la critique du paralogisme de la simplicité, Kant fait la supposition que le moi pensant est soit simple, soit composé, et il pose ensuite la question: est-ce que le psychologue rationnel peut arriver à montrer, en partant de cette supposition, que le moi est une chose simple et immatérielle plutôt que divisible et matérielle? La réponse, dit-il, est négative parce que, d'une part, il n'y a aucune incohérence à dire qu'une pensée qui réunit ensemble plusieurs représentations est pensée par un sujet composé et, de l'autre, on ne connaît pas (de toute façon) la nature du sujet tel qu'il est en soi. Dans cette hypothèse, donc, on suppose que soit le dualisme (cartésien), soit le matérialisme est vrai. Mais on peut faire également une autre hypothèse. Supposons avec le psychologue rationnel, mais cette fois contre le matérialiste, que le moi est une substance indivisible : est-ce qu'il s'ensuit une différence de nature avec les choses matérielles et est-ce que cela prouve que le moi est une substance indestructible? Non, puisque, premièrement, la matière, comme substance composée, n'existe pas en soi, si bien que l'on peut concevoir que le substrat de la matière soit lui aussi simple en lui-même ; et, deuxièmement, même en tant que substance simple, le moi pourrait cesser d'exister autrement que par division (il pourrait disparaître par « consomption»). Une troisième hypothèse est aussi possible : admettons que le psychologue rationnel a raison d'affirmer contre le matérialiste que le moi est un être 
pensant en soi. La même question se pose : est-ce que nous serions justifiés à en conclure à une différence de nature avec la matière? Et la réponse est similaire : l'objet transcendantal du sens externe (l'objet extérieur en soi), bien qu'il nous apparaisse comme matériel, pourrait être en lui-même une chose pensante ; on pourrait même imaginer que toute substance est par essence une chose pensante comme le soutiennent les tenants du pneumatisme. Bref, quelle que soit l'hypothèse que l'on concède au psychologue rationnel, celui-ci ne parvient toujours pas à démontrer que le moi est une substance immatérielle. Kant, pour sa part, n'affirme la vérité d'aucune de ces suppositions. Il rejette, comme on l'a vu, la première hypothèse : puisque le moi ne peut être une substance composée, alors il est faux de dire qu'il est soit simple, soit composé. Il rejette également la troisième, d'après le passage cité ci-dessus, car le moi n'est pas un être pensant en lui-même. Cependant, comme je l'ai noté plus haut, il ne rejette pas, semble$t$-il, la seconde hypothèse : la conclusion de l'argument contre le matérialisme montre que sa position reste entièrement compatible avec la supposition que le moi est une substance simple en soi.

Par ailleurs, sa critique du dualisme cartésien et du matérialisme ne réfute pas d'autres formes de dualisme et de monisme. Il demeure tout à fait possible d'admettre l'existence, comme constituants ultimes de la réalité, d'un seul genre de substance, ou de deux genres de substance ou, peut-être, de plus de deux. En particulier, un individu humain pourrait être formé de deux substances, comme le pense le dualiste, des substances d'une nature différente, et il se pourrait aussi, comme le pense le matérialiste, qu'il ne soit constitué que d'une seule substance; néanmoins, nous ne pouvons pas savoir laquelle de ces hypothèses est vraie et tout ce dont nous pouvons être certains, c'est qu'aucune de ces substances n'est en soi un être pensant ou une chose matérielle. Un corollaire de son point de vue, semble-t-il, est qu'il devient impossible de donner une explication physico-chimique (ou neurophysiologique) des fonctionnements de l'esprit ou des processus mentaux d'une personne. Expliquer en termes physiques ou chimiques les opérations de l'esprit supposerait en effet que, lorsque nous parlons de tel ou tel état ou processus de l'esprit, nous nous référons en fait simplement à un état ou processus ayant lieu dans le corps de la personne, par exemple à une activité de son 
cerveau. Et ceci voudrait dire que l'esprit et le corps d'un individu sont une seule et même chose ou, autrement dit, que l'objet transcendantal du sens interne (le moi en soi) est identique à l'objet transcendantal du sens externe. Mais puisque, pour Kant, nous ne pouvons pas déterminer si une personne c'est une seule substance et non pas plutôt deux, toute tentative d'explication physique des processus mentaux devient pour le moins problématique dans la mesure où elle présuppose justement une telle identité entre une personne et son corps.

En résumé, j’ai voulu développer et rendre plus explicites dans ce qui précède les différentes objections que fait Kant à l'endroit du matérialisme et de la psychologie rationnelle. J'ai essayé de montrer en particulier quel rôle joue dans la critique des deux doctrines la thèse de l'idéalité des phénomènes, laquelle est une conséquence de son idéalisme transcendantal. L'idéalité des phénomènes du sens externe, c'est-à-dire le caractère subjectif des propriétés des objets qui nous les font apparaître comme matériels et dans l'espace, entraine le rejet du matérialisme ; l'idéalité des phénomènes du sens interne, le fait que les pensées ne soient pas des propriétés du moi en soi et que celui-ci apparaisse seulement comme une chose qui pense, implique le rejet du dualisme cartésien. Contre le matérialiste, Kant soutient que rien n'est par nature matériel et, contre le dualiste cartésien, que le moi n'est pas une chose dont l'essence réside dans la pensée. Ici, comme ailleurs dans la Critique de la raison pure, on peut dire sans doute que l'idéalisme transcendantal reste une des thèses centrales de son épistémologie.

Département de philosopbie,

C.E.G.E.P. de Saint-Hyacinthe 